\title{
Effects of configuration mixing in heavy-ion elastic scattering
}

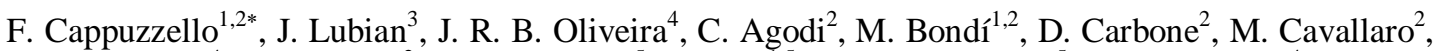
L. C. Chamon ${ }^{4}$ A. Cunsolo ${ }^{2}$, M. De Napoli ${ }^{5}$, A. Foti ${ }^{1,5}$, V. Nunes Garcia ${ }^{5}$, L. R. Gasques ${ }^{4}$, P. R. S. Gomes $^{3}$, R. Linares ${ }^{3}$, D. Nicolosi ${ }^{1,2}$, B. Paes ${ }^{5}$, S. Tropea ${ }^{1,2}$

${ }^{1}$ Dipartimento di Fisica e Astronomia Università di Catania, via S. Sofia 64, I-95125, Catania, Italy

${ }^{2}$ INFN, Laboratori Nazionali del Sud, via S. Sofia, 62, I-95125, Catania, Italy

${ }^{3}$ Instituto de Física da Universidade Federal Fluminense, Av. Litorânea s/n, Niterói, RJ, 24210-340, Brazil

${ }^{4}$ Departamento de Física Nuclear, Instituto de Física da Universidade de São Paulo, Caixa Postal 66318, São Paulo, SP, 05315-970, Brazil

${ }^{5}$ INFN-Sez. Catania, via S. Sofia 64, I-95125, Catania, Italy

\begin{abstract}
A theoretical study of the influence of configuration mixing on elastic scattering cross section is performed for the system ${ }^{16} \mathrm{O}+{ }^{27} \mathrm{Al}$ at $100 \mathrm{MeV}$. A simple twostate model space, including the ${ }^{27} \mathrm{Al} 5 / 2^{+}$ground and $5 / 2^{+}$excited state at $2.73 \mathrm{MeV}$, is used in the coupled channel equations. The results indicate that even a weak degree of mixing is able to sizeably affect the elastic cross section, determining mainly a damping of Fraunhofer oscillations, as observed in the experiments.
\end{abstract}

\section{Introduction}

Recently high precision elastic and inelastic angular distributions have been measured for the ${ }^{16} \mathrm{O}+$ ${ }^{27} \mathrm{Al}$ system at an incident energy of $100 \mathrm{MeV}$ [1]. The experiment was performed at the INFN-LNS laboratory in Catania using the large acceptance magnetic spectrometer MAGNEX [2-4]. The data analysis, based on parameter-free Coupled Channel (CC) calculations [5] and the São Paulo double folding potential, (SPP) [6] has confirmed the formation of a nuclear rainbow [7]. The failure of Optical Model (OM) calculations with the same optical potential in reproducing the measured cross sections reveals the crucial role of inelastic couplings in the rainbow formation.

In the CC calculations a model space including the target excitation of the $1 / 2^{+}, 3 / 2^{+}, 5 / 2^{+}, 7 / 2^{+}$and $9 / 2^{+}$multiplet of states, built by the weak coupling of ${ }^{28} \mathrm{Si}\left(2^{+}\right)$rotation with the $1 \mathrm{~d} 5 / 2$ proton hole $\left|\left[{ }^{28} \operatorname{Si}\left(2^{+}\right) \otimes(1 d 5 / 2)_{\pi}^{-1}\right]\right\rangle$, was considered. Other excited states of the target and the projectile were found to have a negligible effect. However the possible mixing of the $\left|\left[{ }^{28} \operatorname{Si}\left(0^{+}\right) \otimes(1 d 5 / 2)_{\pi}^{-1}\right]\right\rangle$ and $\left|\left[{ }^{28} \operatorname{Si}\left(2^{+}\right) \otimes(1 d 5 / 2)_{\pi}^{-1}\right]\right\rangle$ configuration in the ${ }^{27} \mathrm{Al} 5 / 2^{+}$ground state and the $5 / 2^{+}$member of the multiplet was not considered. This mixing is known to sensibly reduce the inelastic cross section of the $5 / 2^{+}$excited state in $\mathrm{p}+{ }^{27} \mathrm{Al}$ scattering [8]. Both the $\mathrm{CC}$ and the $\mathrm{OM}$ calculations predict very similar Fraunhofer oscillations due to the interference of near-far side components of the scattering amplitude. Despite observed in the experimental results, the amplitude of the calculated oscillations is

\footnotetext{
* Corresponding author : cappuzzello@lns.infn.it
} 
larger than the measured one [5] even performing the convolution with the experimental angular resolution. Recent partial wave analyses with low angular momentum cut-off have not allowed to find the reason of this damping [9].

In the present work, an analysis in terms of realistic configuration mixing of the ${ }^{27} \mathrm{Al} 5 / 2^{+}$ground and the $5 / 2^{+}$excited state at $2.73 \mathrm{MeV}$ is proposed in order to explain the phenomenon of the damping of Fraunhofer oscillations.

\section{The mixing model}

The basic idea of our proposed approach was to introduce more realistic wave functions for the ${ }^{27} \mathrm{Al}$ $5 / 2^{+}$states as follows:

$\left\{\begin{array}{l}\left|{ }^{27} A l\left(5 / 2^{+}\right)_{0}\right\rangle=\mathrm{a}\left|\left[{ }^{28} \operatorname{Si}\left(0^{+}\right) \otimes(1 d 5 / 2)_{\pi}^{-1}\right]\right\rangle-\sqrt{1-a^{2}}\left|\left[{ }^{28} \operatorname{Si}\left(2^{+}\right) \otimes(1 d 5 / 2)_{\pi}^{-1}\right]\right\rangle \\ \left|{ }^{27} \operatorname{Al}\left(5 / 2^{+}\right)_{1}\right\rangle=\sqrt{1-a^{2}}\left|\left[{ }^{28} \operatorname{Si}\left(0^{+}\right) \otimes(1 d 5 / 2)_{\pi}^{-1}\right]\right\rangle+a\left|\left[{ }^{28} \operatorname{Si}\left(2^{+}\right) \otimes(1 d 5 / 2)_{\pi}^{-1}\right]\right\rangle\end{array}\right.$

where $a$ indicates the spectroscopic amplitude of the leading configuration in each state.

In the coupled channel calculations the different couplings between the components of the two state wave functions were explicitly accounted for. The SPP potential was used as in ref. [5], while the spectroscopic amplitudes were taken from shell model and experimental data [10] and are compatible with those used in ref. [9].

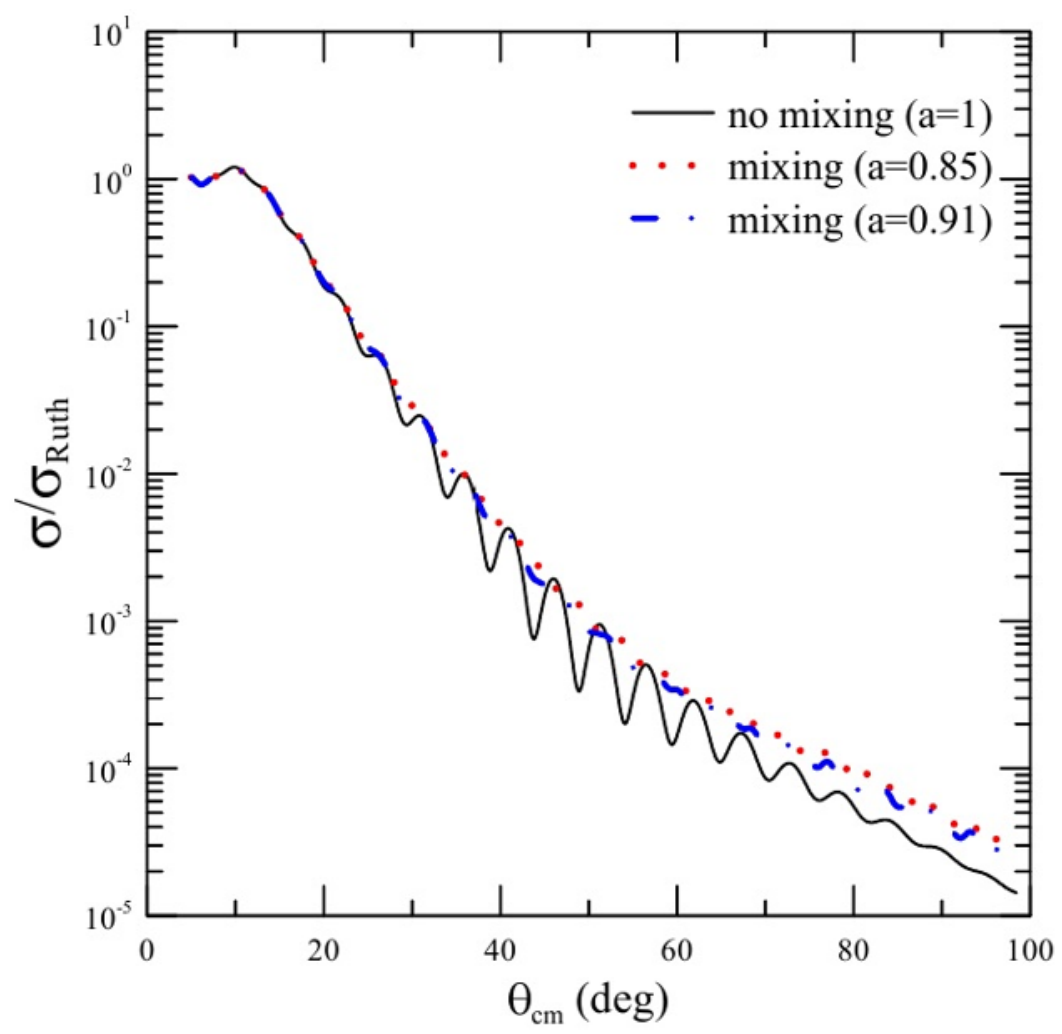

Fig.1 Cross section of the ${ }^{16} \mathrm{O}+{ }^{27} \mathrm{Al}$ elastic scattering at $100 \mathrm{MeV}$ incident energy. The different curves refer to different level of configuration mixing (see text). 
Fig. 1 shows the results of the calculations with and without mixing. The main effect is the damping of the oscillation pattern. There is also an enhancement of the cross section at backward angles. The mixing model introduces matrix elements in the elastic channel that are normally present in the inelastic channel. The damping is thus expected since elastic and inelastic cross sections in a pure (not mixed) model oscillate in opposition of phase [5], as observed in the experiment [1,5]. The surprising result is that even a small configuration mixing can have a large effect, which makes it an important ingredient for future analyses of the elastic cross section. Calculations with the inclusion of the mixing in the full $\left|\left[{ }^{28} \operatorname{Si}\left(2^{+}\right) \otimes(1 d 5 / 2)_{\pi}^{-1}\right]\right\rangle$ model space are under way.

\section{References}

1. M. Cavallaro et al., Nucl. Instr. and Meth. A 648, 46 (2011).

2. F. Cappuzzello et al., MAGNEX: an innovative large acceptance spectrometer for nuclear reaction studies in: Magnets: Types, Uses and Safety, Nova Publisher Inc., New York, 2011, pp 1-63.

3. M. Cavallaro, et al., Eur. Phys. J A 48, 59 (2012).

4. F. Cappuzzello, et al., Nucl. Instr. Meth. A 638, 74 (2011).

5. D. Pereira, J. Lubian, J.R.B. Oliveira, L.C. Chamon, Phys. Lett. B 670, 330 (2009).

6. L.C. Chamon et al., Phys. Rev. Lett. 79, 5218 (1997).

7. D. Pereira et al., Phys. Lett. B 710, 426 (2012).

8. G.M. Crawley, G.T. Garvey, Phys. Lett. 19 (1965) 228, and references therein.

9. J.R.B.de Oliveira et al. J. Phys. G: Nucl. Part. Phys. 40 (2013) 105101.

10. B. H. Wildenthal and J. B. McGrory Phys. Rev. C 7, 714 (1973), and references therein. 
